\title{
The research of vibrations of the beam with the attached system of mass-spring-damper using functions Tymoshenko
}

\author{
Ivanov Pavel $^{1, *}$ \\ ${ }^{1}$ Moscow State University of Civil Engineering, Yaroslavskoye Shosse, 26, Moscow, 129337, \\ Russian Federation
}

\begin{abstract}
In the article [1], is considered a classic method for analyze the dynamics of the loaded beam, as a result leading to the solution of a system of transcendental equations for the eigenvalues. In this paper, shall show that perhaps the solution using functions Tymoshenko arising in the analysis of the free beam vibrations. As a result, the problem will be reduced to infinite dimensional system of differential equations satisfying the conditions for the approximate solution.
\end{abstract}

\section{Introduction}

The study of the beam vibrations continues to be actual over the years and finds wide application in engineering practice. The authors [4-6] studied this problem is performed analysis to find eigenfrequencies and eigenmodes. This approach is useful when considering the free vibrations of the beam. If mass or spring-mass attach to a beam, having difficulty with the calculation of eigenfrequencies. In this paper, we will show how, without changing the system of eigenfunctions, explore the fluctuations in the beam with a system of mass-spring-damper.

\section{Equations of motion}

Consider the free vibrations of the simply-supported uniform beam (length $l$, linear density $\rho$, stiffness $E I$ and area of cross section $F$ ) with system of mass-spring-damper on на interior point $x_{0}$ (Fig. 1.). The stiffness of the spring $g$, a viscosity damper $c$ and mass weight $M$. For this system is well-known equation of motion [2]:

$$
E I \frac{\partial^{4} U}{\partial x^{4}}=-\rho F \frac{\partial^{2} U}{\partial t^{2}}
$$

where $U(x, t)$ - function of a displacement perpendicular to the axis of the beam.

* Corresponding author: ar1on@mail.ru 


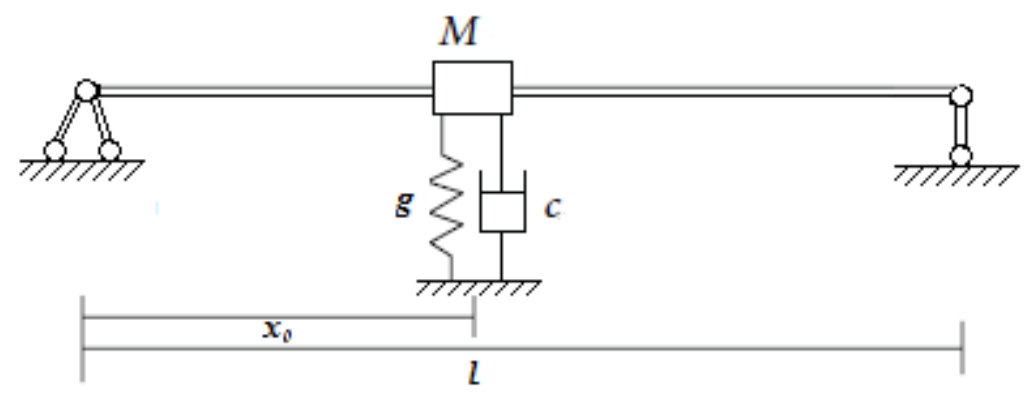

Fig. 1. Beam with system of mass-spring-damper.

The boundary conditions are of the form

$$
\left\{\begin{array} { l } 
{ U | _ { x = 0 } = 0 } \\
{ \frac { \partial ^ { 2 } U } { \partial x ^ { 2 } } | _ { x = 0 } = 0 }
\end{array} \quad \left\{\begin{array}{l}
\left.U\right|_{x=l}=0 \\
\left.\frac{\partial^{2} U}{\partial x^{2}}\right|_{x=l}=0
\end{array}\right.\right.
$$

Influence of a system of mass-spring-damper is determined by the coupling conditions at the point $x_{0}$ :

1) Continuity condition: $\left.U\right|_{x=x_{0}-0}=\left.U\right|_{x=x_{0}+0}$;

2) Conditions smoothness: $\left.\frac{\partial U}{\partial x}\right|_{x=x_{0}-0}=\left.\frac{\partial U}{\partial x}\right|_{x=x_{0}+0}$;

3) Equilibrium of moments: $\left.E I \frac{\partial^{2} U}{\partial x^{2}}\right|_{x=x_{0}-0}=\left.E I \frac{\partial^{2} U}{\partial x^{2}}\right|_{x=x_{0}+0}$;

4) Equilibrium of forces:

$$
E I\left(\left.\frac{\partial^{3} U}{\partial x^{3}}\right|_{x=x_{0}-0}-\left.\frac{\partial^{3} U}{\partial x^{3}}\right|_{x=x_{0}+0}\right)=\left.M \frac{\partial^{2} U}{\partial t^{2}}\right|_{x=x_{0}}+\left.c \frac{\partial U}{\partial t}\right|_{x=x_{0}}+\left.g U\right|_{x=x_{0}} .
$$

\section{The solution using functions Tymoshenko}

Apply the method of separating variables, i.e. $U(x, t)=X(x) \cdot T(t)$.

If consider only (1) and (2), the eigenfunctions would constitute functions Tymoshenko:

$$
X_{n}(x)=\cosh k_{n} x-\cos k_{n} x-\frac{\cosh k_{n} l-\cos k_{n} l}{\sinh k_{n} l-\sin k_{n} l}\left(\sinh k_{n} x-\sin k_{n} x\right), \text { where } k_{n}=\frac{\pi n}{l} .
$$

The orthogonality condition

$$
\int_{0}^{l} X_{n}(x) X_{n}(x) d x=\delta_{n m}
$$

where $\delta_{n m}$ - Kronecker delta function.

Let us assume that the solution of the current problem can be represented in the form: 


$$
U(x, t)=\sum_{n=1}^{\infty} a_{n}(t) X_{n}(x)
$$

And also rightly:

$$
\frac{\partial^{4} U}{\partial x^{4}}=\sum_{n=1}^{\infty} b_{n}(t) X_{n}(x)
$$

To reduce the size of the calculations, we agree that strokes or numbers in brackets at the upper right index indicates the order derivatives of $x$, and the points on functions derivative of $t$.

Establish the relation between $\left\{a_{n}(t)\right\}_{n=1}^{\infty}$ и $\left\{b_{n}(t)\right\}_{n=1}^{\infty}$. For this use the representation $a_{n}(t)$, as the Fourier coefficients and 4 times apply integration by parts and taking into account $X_{n}^{(I V)}(x)-k_{n}^{4} \cdot X(x)=0$ :

$$
\begin{gathered}
a_{n}(t)=\int_{0}^{l} U(x, t) X_{n}(x) d x=\frac{1}{k_{n}^{4}} \int_{0}^{l} U(x, t) X_{n}^{(I V)}(x) d x=\frac{1}{k_{n}^{4}}\left(\left.U(x, t) X_{n}^{\prime \prime \prime}(x)\right|_{0} ^{l}-\right. \\
\left.-\int_{0}^{l} U^{\prime}(x, t) X_{n}^{\prime \prime \prime}(x) d x\right)=\frac{1}{k_{n}^{4}}\left(\left.U(x, t) X_{n}^{\prime \prime \prime}(x)\right|_{0} ^{l}-\left.U^{\prime}(x, t) X_{n}^{\prime \prime}(x)\right|_{0} ^{l}+\left.U^{\prime \prime}(x, t) X_{n}^{\prime}(x)\right|_{0} ^{l}-\right. \\
\left.-\int_{0}^{l} U^{\prime \prime \prime}(x, t) X_{n}^{\prime}(x) d x\right)=\frac{1}{k_{n}^{4}}\left(\left.U(x, t) X_{n}^{\prime \prime \prime}(x)\right|_{0} ^{l}-\left.U^{\prime}(x, t) X_{n}^{\prime \prime}(x)\right|_{0} ^{l}+\left.U^{\prime \prime}(x, t) X_{n}^{\prime}(x)\right|_{0} ^{l}-\right. \\
\left.-\left(\int_{0}^{c} U^{\prime \prime \prime}(x, t) X_{n}^{\prime}(x) d x+\int_{c}^{l} U^{\prime \prime \prime}(x, t) X_{n}^{\prime}(x) d x\right)\right)=\frac{1}{k_{n}^{4}}\left(\left.U(x, t) X_{n}^{\prime \prime \prime}(x)\right|_{0} ^{l}-\left.U^{\prime}(x, t) X_{n}^{\prime \prime}(x)\right|_{0} ^{l}+\right. \\
\left.+\left.U^{\prime \prime}(x, t) X_{n}^{\prime}(x)\right|_{0} ^{l}-\left.U^{\prime \prime \prime}(x, t) X_{n}(x)\right|_{0} ^{l}+\left.U^{\prime \prime \prime}(x, t) X_{n}(x)\right|_{x_{0}-0} ^{x_{0}+0}+\int_{0}^{l} U^{(I V)}(x, t) X_{n}(x) d x\right) .
\end{gathered}
$$

In accordance with the boundary conditions, the first four expressions in brackets are zero. We obtain

$$
a_{n}(t)=\frac{1}{k_{n}^{4}}\left(\left(U^{\prime \prime \prime}\left(x_{0}+0, t\right)-U^{\prime \prime \prime}\left(x_{0}-0, t\right)\right) X_{n}\left(x_{0}\right)+\int_{0}^{l} U^{(I V)}(x, t) X_{n}(x) d x\right) .
$$

As can be seen, the integral in the brackets is the coefficient $b_{n}(t)$.

From (4) follows that

$$
\dot{U}(x, t)=\sum_{n=1}^{\infty} \dot{a}_{n}(t) X_{n}(x) \quad \ddot{U}(x, t)=\sum_{n=1}^{\infty} \ddot{a}_{n}(t) X_{n}(x)
$$

Substitute the expression for $a_{n}(t)$, have 


$$
a_{n}(t)=\frac{1}{k_{n}^{4}}\left(-\frac{1}{E I}\left(\left.M \frac{\partial^{2} U}{\partial t^{2}}\right|_{x=x_{0}}+\left.c \frac{\partial U}{\partial t}\right|_{x=x_{0}}+\left.g U\right|_{x=x_{0}}\right) X_{n}\left(x_{0}\right)+b_{n}(t)\right) .
$$

Apply (4) and obtain the final formula

$$
a_{n}(t)=\frac{1}{k_{n}^{4}}\left(-\frac{X_{n}\left(x_{0}\right)}{E I} \sum_{s=1}^{\infty}\left(M \ddot{a}_{s}(t)+c \dot{a}_{s}(t)+g a_{s}(t)\right) X_{s}\left(x_{0}\right)+b_{n}(t)\right)
$$

or

$$
b_{n}(t)=k_{n}^{4} a_{n}(t)+\frac{X_{n}\left(x_{0}\right)}{E I} \sum_{s=1}^{\infty}\left(M \ddot{a}_{s}(t)+c \dot{a}_{s}(t)+g a_{s}(t)\right) X_{s}\left(x_{0}\right)
$$

The formula is clearly seen the main difference of this method from the classic separation of variables - additional load is taken into account in the Fourier coefficients, rather than in orthogonal conditions eigenfunctions. Also note that when $M=k=c=0$, obtain $b_{n}(t)=k_{n}^{4} a_{n}(t)$, which is consistent with the results for the free vibration.

Write the equation (1) substituting (4) and (5)

$$
\begin{array}{r}
E I \sum_{n=1}^{\infty} b_{n}(t) X_{n}(x)=-\rho F \sum_{n=1}^{\infty} \ddot{a}(t) X_{n}(x) \\
\sum_{n=1}^{\infty}\left(E I \cdot b_{n}(t)+\rho F \cdot \ddot{a}_{n}(t)\right) X_{n}(x)=0 . \\
E I \cdot b_{n}(t)+\rho F \cdot \ddot{a}_{n}(t)=0, \text { где } n=\overline{1, \infty} .
\end{array}
$$

Using resulting formula (6) in the last equation

$$
k_{n}^{4} E I a_{n}(t)+X_{n}\left(x_{0}\right) \sum_{s=1}^{\infty}\left(M \ddot{a}_{s}(t)+c \dot{a}_{s}(t)+g a_{s}(t)\right) X_{s}\left(x_{0}\right)+\rho F \cdot \ddot{a}_{n}(t)=0, \text { где } n=\overline{1, \infty}
$$

or

$$
\begin{gathered}
\left(M X_{n}^{2}\left(x_{0}\right)+\rho F\right) \ddot{a}_{n}(t)+c X_{n}^{2}\left(x_{0}\right) \dot{a}_{n}(t)+\left(k_{n}^{4} E I+g X_{n}^{2}\left(x_{0}\right)\right) a_{n}(t)= \\
\quad=-X_{n}\left(x_{0}\right) \sum_{\substack{s=1 \\
s \neq n}}^{\infty}\left(M \ddot{a}_{s}(t)+k \dot{a}_{s}(t)+c a_{s}(t)\right) X_{s}\left(x_{0}\right), \text { где } n=\overline{1, \infty} .
\end{gathered}
$$

For practical application, the system can be approximately solved using numerical methods and reduction method [3], because $\left\{X_{n}^{2}\left(x_{0}\right)\right\}_{n=1}^{\infty}$ limited and $\lim _{n \rightarrow \infty} a_{n}(t)=0$.

\section{Conclusion}

In this article, we have been shown a method which does not require calculation of eigenfunctions and eigenfrequencies, and reduces the problem to the infinite dimensional 
system of differential equations. This system can easily be solved using numerical methods and method of reduction, since It satisfies the necessary conditions.

Since the solution uses functions Tymoshenko system it makes it easier the problem of convergence of Fourier series, and also allows a comparative analysis with non-loaded beam.

It is worth noting that all of the solution was performed for free-supported beam, but it is similar to any combination of the classic fixings. Enough to take corresponding functions Tymoshenko. This solution can be generalized to multiple systems of mass-spring-damper.

\section{References}

1. O. Barry, D.C.D. Oguamanam, J.W. Zu, Shock and Vibration, 2014, 9 (2014)

2. S.P. Tymoshenko, Vibration problems in engineering, Mashinostroenie (1985)

3. L.V. Kantorovich, G.P. Akilov, Functional Analysis, Phys.-Mat. Lit. (1962)

4. G. Chen, L. Qian, Q. Yin, Shock and Vibration, 2014, 12 (2014)

5. H.-Y. Lin, Y.-C. Tsai, Journal of Sound and Vibration, 302, 442-456 (2007)

6. M.H. Ghayesh, F. Alijani, M.A. Darabi, Journal of mechanical Science and Technology, 25, 1915-1923 (2011) 